\title{
Apoptosis-sensitizing activity of birinapant in head and neck squamous cell carcinoma cell lines
}

\author{
ROMAN C. BRANDS ${ }^{1,2}$, MARIO J.J. SCHEURER ${ }^{1}$, STEFAN HARTMANN $^{1,3}$, AXEL SEHER ${ }^{1}$, \\ ALEXANDER C. KÜBLER ${ }^{1}$ and URS D.A. MÜLLER-RICHTER ${ }^{1}$ \\ ${ }^{1}$ Department of Oral and Maxillofacial Plastic Surgery, University Hospital Würzburg, D-97070 Würzburg; \\ ${ }^{2}$ Comprehensive Cancer Center Mainfranken, University Hospital Würzburg, D-97080 Würzburg; \\ ${ }^{3}$ Interdisciplinary Center for Clinical Research, University Hospital Würzburg, D-97070 Würzburg, Germany
}

Received July 24, 2017; Accepted October 20, 2017

DOI: $10.3892 / \mathrm{ol} .2018 .7783$

\begin{abstract}
Inhibitor of apoptosis proteins, which are overexpressed in head and neck squamous cell carcinoma (HNSCC), may cause therapeutic resistance. Using SMAC mimetic compounds, including birinapant, to degrade and/or inhibit these proteins and sensitize apoptosis may enhance therapies in HNSCC. Fas expression was analyzed in nine HNSCC cell lines and one keratinocyte cell line via flow cytometry. These cell lines were treated with Fas ligand-Fc (FasL) and birinapant, a bivalent SMAC mimetic, in mono and combination therapies. Cytotoxicity was measured using a crystal violet assay. Annexin V assay was performed for detection of apoptosis. The treatment efficacy of mono and combination therapies was statistically analyzed. Nonlinear regression analysis was performed to determine the inhibitory concentration $\left(\mathrm{IC}_{10}\right)$ of birinapant. Fas expression was detected in each cell line tested. Mono treatment with FasL revealed minor to no apoptotic effects in the majority of the cell lines. Crystal violet and Annexin V staining revealed increased apoptosis rates for all cell lines following incubation with birinapant in mono treatment. Combination treatment with FasL and birinapant $\left(\mathrm{IC}_{10}\right)$ revealed additional and synergistic effects in eight out of the ten cell lines. To the best of our knowledge, the present study provided the first evidence of the apoptosis-sensitizing activity of combination treatment with FasL and birinapant in HNSCC cell lines.
\end{abstract}

\section{Introduction}

Head and neck squamous cell carcinoma (HNSCC), with 600,000 new cases/year, represents the sixth most common

Correspondence to: Dr Roman C. Brands, Department of Oral and Maxillofacial Plastic Surgery, University Hospital Würzburg, 2 Pleicherwall, D-97070 Würzburg, Germany

E-mail: brands_r@ukw.de

Key words: head and neck squamous cell carcinoma, apoptosis, second mitochondria-derived activator of caspase, inhibitor of apoptosis tumor entity worldwide (1). According to the Robert Koch Institute (Berlin, Germany), 13,000 new cases were diagnosed in Germany in 2012 (2). With respect to the treatment of HNSCC, comprising surgery, radiation and chemotherapy, the 5-year survival rate has not markedly changed in recent decades (1,3). Pembrolizumab and nivolumab, 'checkpoint inhibitors' that exhibit antitumor efficacy in other tumor entities, were recently approved by the US Food and Drug Administration as the only treatment innovations in the past decade (4).

Apoptosis is of fundamental importance to normal and abnormal cells. In numerous diseases, including HNSCC, programmed cell death is impaired and cells manage to evade apoptosis (5). There are two main apoptotic pathways. The extrinsic pathway is activated from the direct cellular environment by extracellular ligands that bind tumor necrosis factor receptor-superfamily (TNFR) members, including Fas or TNFR1. The intrinsic pathway of apoptosis is induced in response to nuclear stress, resulting in the release of second mitochondria-derived activator of caspase (SMAC)/direct IAP-binding protein with low PI, among other factors from the mitochondria, and the formation of the 'apoptosome' (6). Caspase cascades are activated via the intrinsic and extrinsic pathways and result in apoptosis. The so-called inhibitor of apoptosis proteins (IAPs) comprise $\mathrm{X}$ chromosome-linked IAP (XIAP), cellular IAP1 (cIAP1) and cIAP2, among others. These proteins counteract apoptosis by directly binding and inhibiting caspases and activating the anti-apoptotic nuclear factor (NF) $-\kappa B$ pathway (7). Potent antagonists of IAPs, including SMAC, eliminate their inhibitory effect by directly binding these proteins $(8,9)$. 'SMAC mimetics', synthetic SMAC analogs, including birinapant, which degrades cIAP1/2 and inhibits XIAP, result in caspase activation by interacting with IAPs and blocking their signaling pathways (6). Overexpressed in solid tumors of the head and neck, IAPs serve an important function as predictors of the cisplatin response and patient prognosis $(10,11)$. In a preclinical setting, Matzinger et al (12) demonstrated the radiosensitizing activity of the SMAC mimetic Debio 1143. Eytan et al (13) described the antitumor activity of birinapant alone or in combination with tumor necrosis factor- $\alpha$ (TNF- $\alpha)$, TNF-related apoptosis inducing ligand and docetaxel in preclinical models of 
HNSCC. Furthermore, Sun et al (10) demonstrated the chemosensitizing activity of SMAC in combination with different anticancer agents in vitro. Recently, several early clinical trials have attempted to evaluate the efficacy of SMAC mimetics alone or in combination with other anticancer drugs (14).

Based on this evidence, the aim of the present study was to assess the treatment efficacy of the SMAC mimetic, birinapant, a bivalent SMAC mimetic targeting cIAP1/2 and XIAP with Fas ligand (FasL), a Fas-binding ligand, resulting in its trimerization and activation of the extrinsic pathway. To the best of our knowledge, the present study is the first to evaluate this combination treatment in HNSCC cell lines.

\section{Materials and methods}

Cell lines. As previously described, the cell lines were cultured at $37^{\circ} \mathrm{C}$ in a humidified atmosphere of $5 \% \mathrm{CO}_{2} / 95 \%$ air, and the medium was changed 2-3 times/week $(15,16)$. Certain cell lines (PCI-1, PCI-9, PCI-13, PCI-52 and PCI-68) were established at the Cancer Institute of the University of Pittsburgh (Pittsburgh, PA, USA), while the other cell lines (Detroit 562, FaDu, SCC9 and SCC25) were purchased from the American Type Culture Collection (ATCC; Manassas, VA, USA). The cells were cultured at $37^{\circ} \mathrm{C}$ in Dulbecco's modified Eagle's medium supplemented with $10 \%$ fetal calf serum, and $1 \%$ penicillin/streptomycin (all from Gibco; Thermo Fisher Scientific, Inc., Waltham, MA, USA) and 1\% glutamine (Biochrom KG, Berlin, Germany).

Drugs. Birinapant (Medivir AB, Huddinge, Sweden) was stored according to the manufacturer's protocol. Affinity chromatography with anti-FLAG M2 agarose beads (Sigma-Aldrich; Merck KGaA, Darmstadt, Germany) was used to purify human recombinant FLAG-tagged soluble Fc-Flag-FasL from the supernatants of 293 cells transfected with the corresponding expression plasmid. FasL (FasL-Fc is referred to as FasL throughout the paper) concentrations $(50,25,12,6,3$ and $1 \mathrm{ng} / \mu \mathrm{l})$ and birinapant concentrations $(200,100,50,25,12,6,3,1$ and $0.5 \mu \mathrm{M})$ were derived from $\log _{2}$ dilutions. These findings were based on multiple experiments, the results of which were consistent with the literature $(13,17)$. For experiments using combination treatment with FasL and birinapant, the aforementioned $\log _{2}$ dilution of FasL and a constant concentration $\left(\mathrm{IC}_{10}\right)$ of birinapant were used for each cell line (Table I). Subsequently, the cultures were incubated at $37^{\circ} \mathrm{C}$ for $72 \mathrm{~h}$.

FACS analysis and Annexin V assay. Cell surface Fas receptor expression was measured via flow cytometry (FACS). Anti-cluster of differentiation 95 (CD95)-phycoerythrin (PE) mouse anti-human (BD Biosciences, Franklin Lakes, NJ, USA) antibody was used to label Fas receptors. Immunoglobulin G2B isotype control PE antibody (R\&D Systems, Inc., Minneapolis, MN, USA) was used as a negative control. The reagents were stored and used according to the manufacturer's instructions.

For the analysis of apoptosis, the cells were double-stained with Annexin $\mathrm{V}$ and 7-AAD using the Annexin V PE Apoptosis Detection kit (eBioscience Inc.; Thermo Fisher
Table I. Name and origin of the ten cell lines used in the present study.

\begin{tabular}{|c|c|}
\hline Cell line & Description \\
\hline PCI-1 & $\begin{array}{l}\text { Laryngeal carcinoma of the glottis of a male } \\
\text { patient }\end{array}$ \\
\hline PCI-9 & $\begin{array}{l}\text { Primary carcinoma of the tongue of a male } \\
\text { patient }\end{array}$ \\
\hline PCI-13 & $\begin{array}{l}\text { Carcinoma of the retromolar triangle of a male } \\
\text { patient }\end{array}$ \\
\hline PCI-52 & $\begin{array}{l}\text { Primary carcinoma of the aryepiglottic fold of } \\
\text { a male patient }\end{array}$ \\
\hline PCI-68 & Primary tongue carcinoma of a male patient \\
\hline $\mathrm{FaDu}$ & Pharyngeal carcinoma of a female patient \\
\hline Detroit 562 & Hypopharyngeal carcinoma of a male patient \\
\hline SCC9 & Carcinoma of the tongue of a male patient \\
\hline SCC25 & Carcinoma of the tongue of a male patient \\
\hline $\mathrm{HaCaT}$ & Keratinocytes \\
\hline
\end{tabular}

Scientific, Inc.) according to the manufacturer's instructions. The cells were analyzed using a BD FACSCalibur platform with CellQuest Pro 5.1 software (BD Biosciences). The results are provided as dot plots with the relative signal intensity of Annexin V vs. 7-AAD. Unstained cells were used as a negative control, and the dot plots were subdivided into four quadrants. The graphs were generated using FlowJo software (version 10; FlowJo LLC, Ashland, OR, USA). In these FACS plots, necrotic cells are represented in the upper left quadrant (Q1). Late apoptotic and dead cells are shown in the upper right quadrant (Q2). Early apoptotic cells are represented in the lower right quadrant (Q3), and live cells are shown in the lower left quadrant (Q4). The percentage of the total cell count is expressed in each quadrant.

Crystal violet assay. Cells of each line (PCI-1, PCI-9, PCI-13, PCI-52, PCI-68, Detroit 562, FaDu, SCC9, SCC25 and $\mathrm{HaCaT}$ ) were seeded at 10,000 cells/well at $37^{\circ} \mathrm{C}$ in DMEM supplemented with $10 \%$ fetal calf serum, $1 \%$ penicillin/streptomycin and $1 \%$ glutamine. On the following day, for treatment with a single agent, concentrations of $\log _{2}$ dilution were added. For combination treatment, the $\mathrm{IC}_{10}$ of birinapant and $\log _{2}$ dilution of FasL were added, followed by incubation at $37^{\circ} \mathrm{C}$ for $72 \mathrm{~h}$. Subsequently, the medium was removed, and the remaining cells were stained at room temperature for $12 \mathrm{~min}$ with $50 \mu \mathrm{l} /$ well crystal violet solution (1\% crystal violet; Carl Roth $\mathrm{GmbH}$, Karlsruhe, Germany) in $20 \%$ methanol/double-distilled water and subsequently washed several times with distilled water. The plates were then dried for $24 \mathrm{~h}$. The absorbance was measured at $595 \mathrm{~nm}$ using a microplate reader (Tecan Spectra Rainbow microplate reader; Tecan Deutschland GmbH, Crailsheim, Germany). All experiments were performed in triplicate, and the mean was calculated from at least three independent experiments.

Statistical analysis. Statistical analysis was performed using Prism (version 6.04; GraphPad Software, Inc., La Jolla, CA, USA) and Microsoft Excel 2016 software (Microsoft 

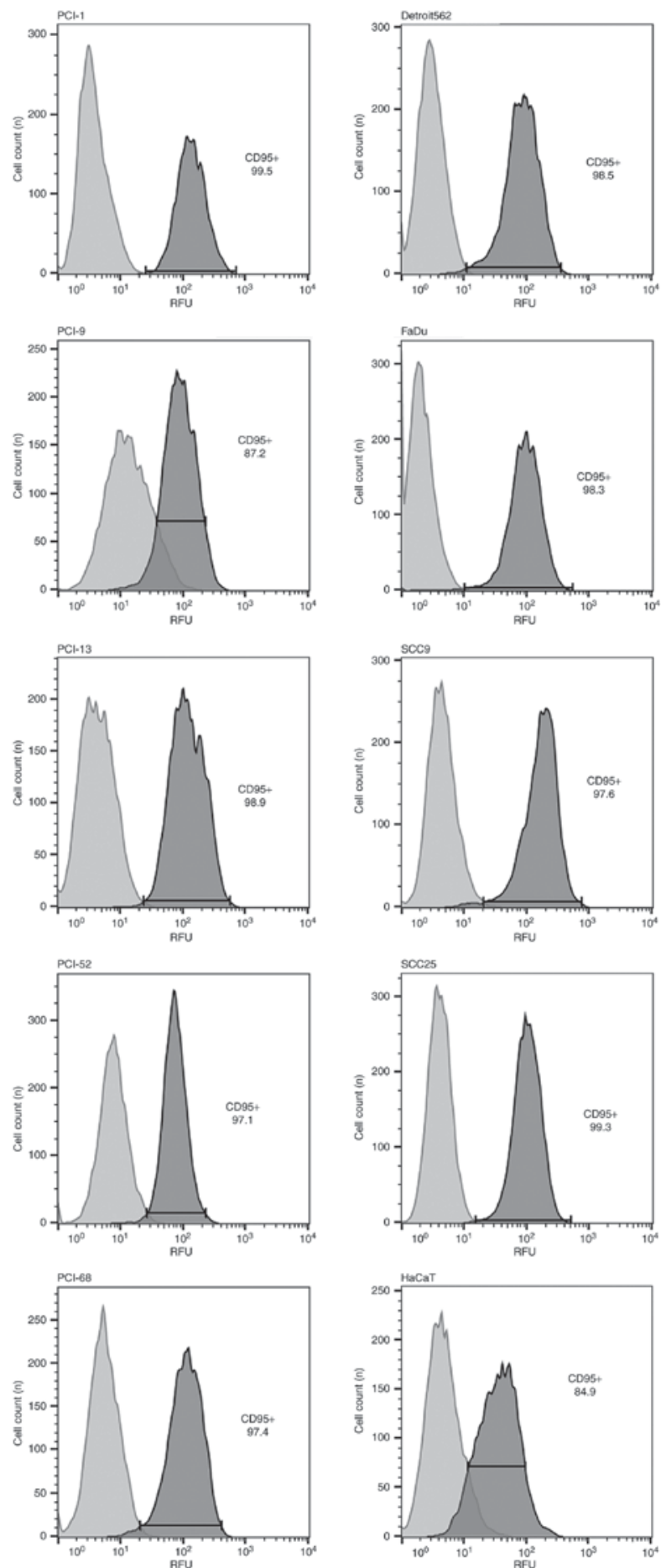

Figure 1. Results of flow cytometry analysis. CD95 expression was detected in all cell lines (PCI-1, PCI-9, PCI-13, PCI52, PCI-68, Detroit 562, FaDu, SCC9, SCC25 and HaCaT). In the histograms, the light gray peak represents the negative isotype control, and the dark gray peak represents the positive control for CD95. CD95, Fas cell surface death receptor.

Corporation, Redmond, WA, USA). P $<0.05$ was considered to indicate a statistically significant difference. The Mann-Whitney test was used to compare mono (FasL) and combination (FasL + birinapant) treatments at different concentrations in each cell line. Using a nonlinear regression analysis, the $\mathrm{IC}_{10}$ was calculated for each cell line. For cell lines for which no $\mathrm{IC}_{10}$ value could be calculated on the basis of their dose-response curves, the concentration that resulted in the first significant $(\mathrm{P}<0.05)$ cell count decrease was defined as the $\mathrm{IC}_{\text {scr }}(\mu \mathrm{M})$ and used for combination treatment. Additive and synergistic effects were distinguished by multiplying the percentage cell number of the mono treatments (FasL and birinapant). If the result was greater than the actual percentage cell count of the combination treatment, the effect was assumed synergistic, if the result was smaller and the combination treatment revealed increased cell count reductions compared with both mono treatments, the effects were assumed additive.

\section{Results}

Fas receptor expression in each cell line. Fas receptor expression was detected in each cell line (PCI-1, PCI-9, PCI-13, PCI-52, PCI-68, Detroit 562, FaDu, SCC9, SCC25 and HaCaT) via flow cytometry (Fig. 1). A dot plot for each cell line was generated to visualize the viable cell fraction. In the histograms, the light gray peak indicates the isotype control, while the dark gray indicates the positive control for Fas receptor expression.

Efficacy of FasL in the cell lines. The majority of the cell lines used in the present study did not exhibit significant reductions in cell count following mono treatment after incubation for $72 \mathrm{~h}$ with FasL (Fig. 2). The cell line PCI-1 responded with a $16.81 \%$ reduction in initial cell number at a FasL concentration of $50 \mathrm{ng} / \mu \mathrm{l}$. The cell lines PCI-9 and PCI-13 exhibited 61.09 and $53.46 \%$ reductions in cell count, respectively at a FasL concentration of $50 \mathrm{ng} / \mu \mathrm{l}$, while the other cell lines (PCI-52, PCI-68, Detroit 562, FaDu, SCC9 and SCC25) exhibited only minimal responses toward the applied concentrations, with cell count reductions ranging from $82.65-100 \%$ of the initial cell number. In addition, the control cell line $\mathrm{HaCaT}$ exhibited only minor effects when treated with FasL (50 ng/ $\mu \mathrm{l})$, with a cell count decrease of $69.98 \%$.

Efficacy of birinapant in the cell lines. The present study also assessed the efficacy of birinapant on the cell lines. A crystal violet assay was used to determine the dose-response curves for mono treatment with birinapant and to establish the respective inhibitory concentrations $\left(\mathrm{IC}_{10} / \mathrm{IC}_{\mathrm{scr}}\right)$ for each cell line for use in combination treatment. The calculated $\mathrm{IC}_{10}$ values were: $18.2 \mu \mathrm{M}$ for PCI-1, 1.3 $\mu \mathrm{M}$ for PCI-9 and $11.5 \mu \mathrm{M}$ for PCI-13. The first concentrations generating a significant cell count reduction, which were subsequently used for combination treatment, were $100 \mu \mathrm{M}$ for PCI-52, $25 \mu \mathrm{M}$ for PCI-68, $50 \mu \mathrm{M}$ for Detroit 562, $25 \mu \mathrm{M}$ for $\mathrm{FaDu}, 1.6 \mu \mathrm{M}$ for SCC9, $1.6 \mu \mathrm{M}$ for SCC25 and 1.6 $\mu \mathrm{M}$ for HaCaT. To confirm the apoptosis-sensitizing activity of birinapant in these cell lines, flow cytometry analysis was performed using an Annexin V assay. The apoptosis assay indicated that the cell lines exhibited mixed responses when exposed to $\mathrm{IC}_{10} / \mathrm{IC}_{\mathrm{scr}}$ concentrations (Fig. 3). While the first image depicts the control, the second image represents the results of the apoptosis assay following incubation for $48 \mathrm{~h}$ with birinapant at the cell line-specific concentrations. Out of ten cell lines, four cell lines (PCI-9, PCI-52, PCI-68 and SCC9) revealed only a slight effect, with an increase in the specific apoptotic rate at a factor of 1.14-1.71 following incubation with $\mathrm{IC}_{10} / \mathrm{IC}_{\text {scr }}$ birinapant. A stronger effect was achieved following the incubation of the cell lines 

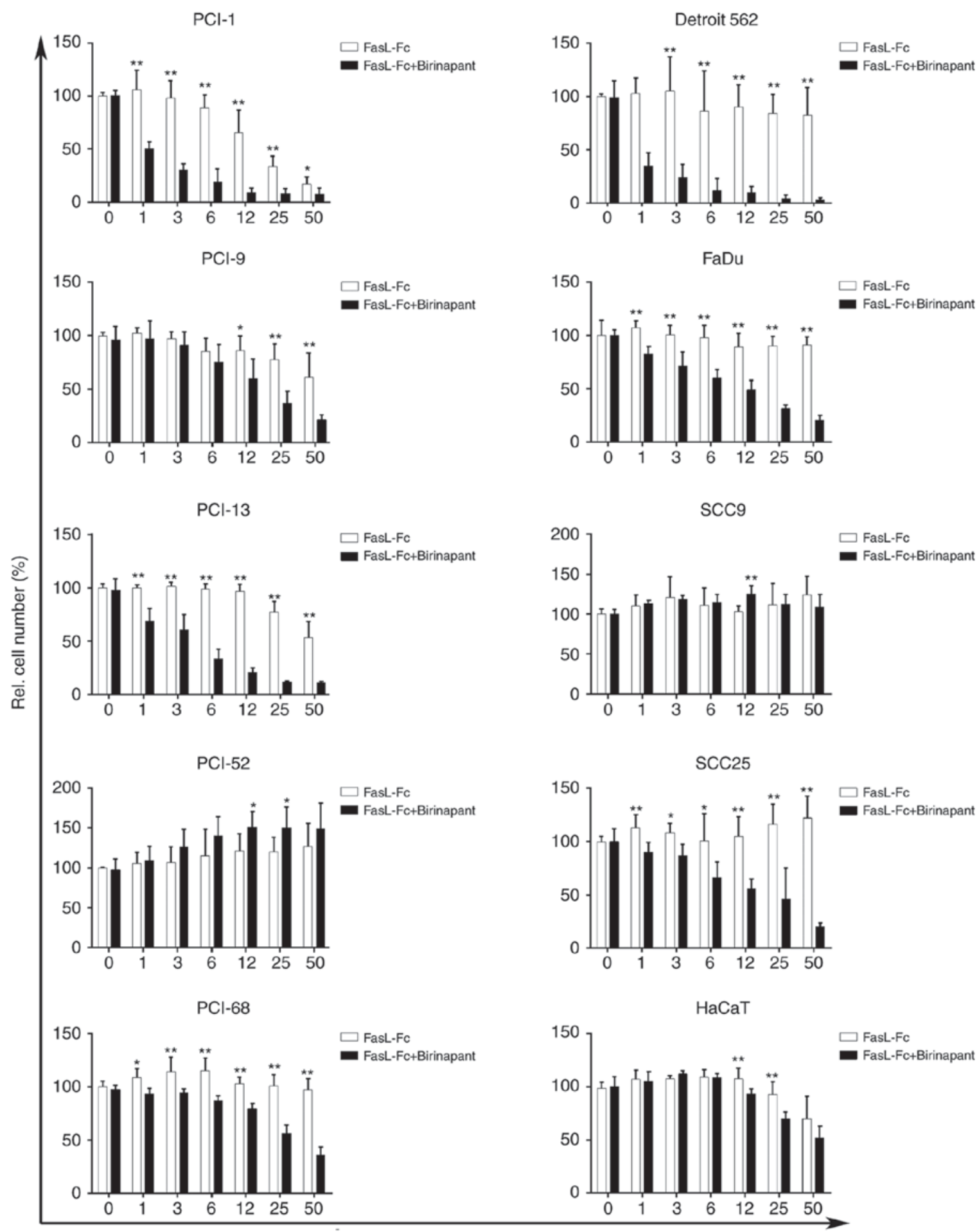

Concentration $(\mathrm{ng} / \mathrm{\mu l})$

Figure 2. Relative cell number (\%), as measured using crystal violet assay. As indicated by the white columns, mono treatment with FasL resulted in a concentration-dependent decrease in relative cell number in PCI-1, PCI-9 and PCI-13 cell lines. The PCI-52, PCI-68, Detroit 562, FaDu, SCC9 and SCC25 cell lines exhibited little to no response toward FasL mono treatment. As indicated by the black columns, treatment with FasL $(\log 2)+$ birinapant $\left(\mathrm{IC}_{10} / \mathrm{IC}_{\mathrm{scr}}\right)$ resulted in a concentration-dependent decrease of the viable cell fraction in eight cell lines (PCI-1, PCI-9, PCI-13, PCI-68, Detroit 562, FaDu, SCC25 and $\mathrm{HaCaT}$ ) with additive and synergistic effects, while two cell lines (PCI-52 and SCC9) did not respond to exposure to FasL and birinapant. "P $\leq 0.05 ;{ }^{* *} \mathrm{P} \leq 0.01$ (corresponding concentrations of mono and combination treatment were compared). FasL, Fas ligand-Fc; rel., relative; $\mathrm{IC}_{10}$, inhibitory concentration of $10 \%$; $\mathrm{IC}_{\mathrm{scr}}$, inhibitory concentration of the first significant cell number reduction.

PCI-1, Detroit 562, FaDu and SCC25 with birinapant, where the apoptotic rate was increased by a factor of 2.21-3.22. In addition, the control cell line HaCaT exhibited a 3.5 -fold increase in apoptotic rate following incubation with $\mathrm{IC}_{10} / \mathrm{IC}_{\text {scr }}$ birinapant. The largest increase in apoptotic rate following birinapant incubation was observed in the cell line PCI-13 (factor of 5.5). All cell lines exhibited a specific increase in apoptotic rate following birinapant treatment. 

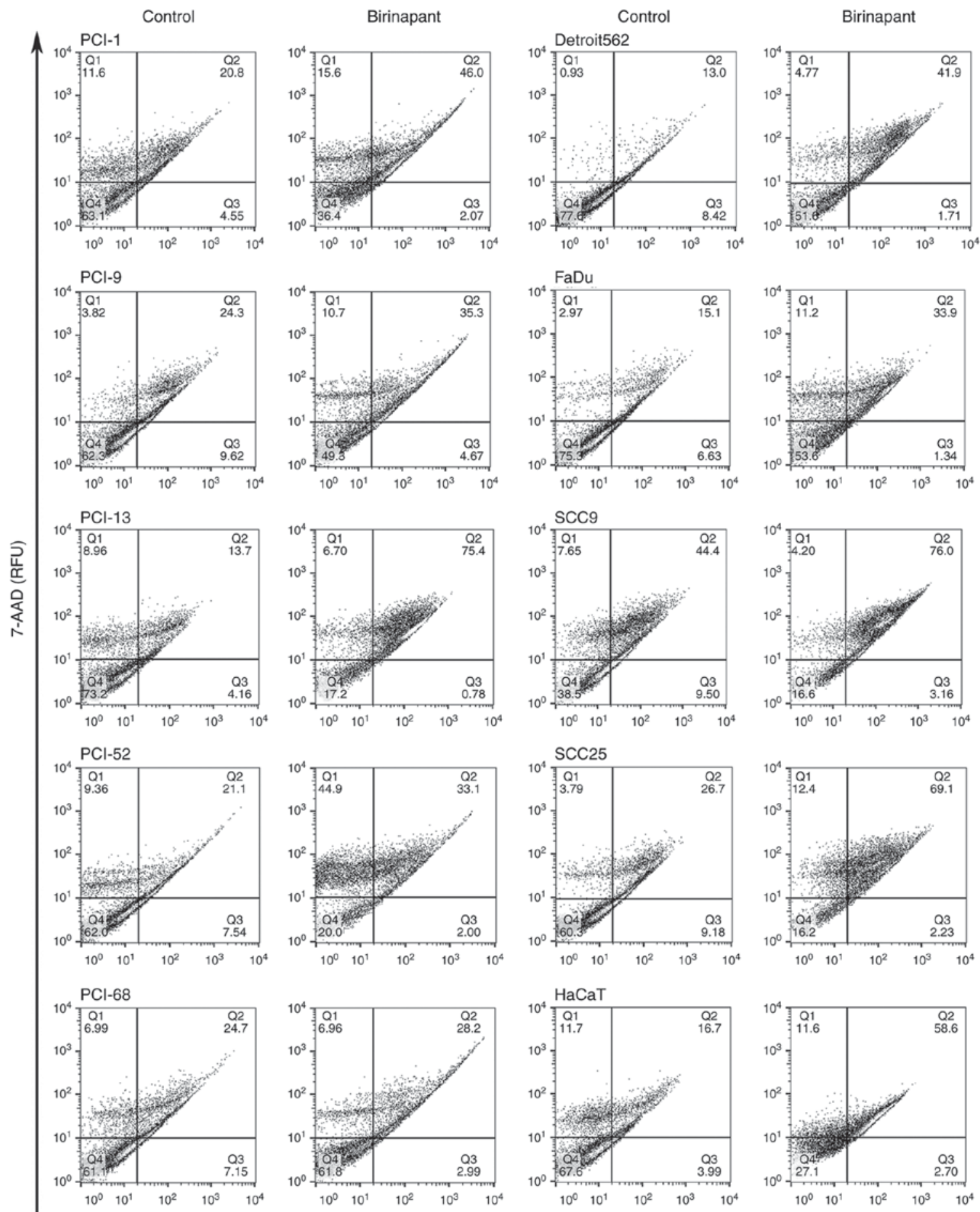

Annexin V (RFU)

Figure 3. Analysis of Annexin V induction using flow cytometry. The figure shows dot plots divided into four quadrants: i) The cells in Q1 were considered necrotic; ii) the cells in Q2 were considered late apoptotic; iii) the cells in Q3 were considered early apoptotic; and iv) the cells in Q4 were considered live. For each cell line, the first image represents the control and the second image reveals results of $\mathrm{FACS}$ analysis following $48 \mathrm{~h}$ of incubation with $\mathrm{IC}_{10} / \mathrm{IC}_{\mathrm{scr}}$ birinapant. All cell lines exhibited a decrease in the number of early apoptotic cells, favoring an increase in the number of late apoptotic cells compared with the number of control cells incubated with birinapant. Q, quadrant; RFU, relative fluorescence unit; $\mathrm{IC}_{10}$, inhibitory concentration of $10 \%$; $\mathrm{IC}_{\mathrm{scr}}$, inhibitory concentration of the first significant cell number reduction.

Apoptosis-sensitizing activity of combination treatment with FasL and birinapant. The crystal violet assay was used to determine the dose-response curves for combination treatment with FasL with an initial concentration of $50 \mathrm{ng} / \mu 1 \log _{2}$ dilution and a constant concentration of birinapant $\left(\mathrm{IC}_{10} / \mathrm{IC}_{\text {scr }}\right.$;
Fig. 2). Mono treatment and combination treatment were plotted against each other. The cell lines PCI-52 and SCC9 did not exhibit significant reductions in cell number following mono or combination treatment. In addition, the use of combination treatment did not reveal any additive or synergistic 
effects compared with mono treatment with FasL, which was supported by the finding in the Annexin V assay with only a slight increase in apoptotic rate (Fig. 3). However, the cell line PCI-9 exhibited a positive effect in response to combination treatment through direct comparison of the different treatment regimens. Additive effects in PCI-9 were observed at concentrations of FasL ranging from 3-50 ng/ $\mu \mathrm{l}$ and $\mathrm{IC}_{10} / \mathrm{IC}_{\text {scr }}$ birinapant, and significant $(\mathrm{P}<0.05)$ reductions in cell number were achieved at a concentration of $12 \mathrm{ng} / \mu \mathrm{l} \mathrm{FasL}$ and $\mathrm{IC}_{10} / \mathrm{IC}_{\text {scr }}$ birinapant in comparison to mono treatment with FasL (12 ng/ $\mu \mathrm{l}$; Fig. 2). The cell line SCC25 exhibited significant reductions in cell number even when exposed to the lowest concentration ( $1 \mathrm{ng} / \mu \mathrm{l}$ ) of FasL and $\mathrm{IC}_{10} / \mathrm{IC}_{\mathrm{scr}}$ birinapant with additive effects, while synergistic effects were achieved at a concentration of $25 \mathrm{ng} / \mu \mathrm{l}$ FasL and $\mathrm{IC}_{10} / \mathrm{IC}_{\text {scr }}$ birinapant, which is also consistent with the findings in the apoptosis assay. The remaining five cell lines (PCI-1, PCI-13, PCI-68, Detroit 562 and FaDu) responded to combination treatment with significant $(\mathrm{P}<0.01$ for PCI-1, PCI-13, Detroit 562 and FaDu, P<0.05 for PCI-68) reductions in cell count when exposed to the lowest concentration $(1 \mathrm{ng} / \mu \mathrm{l})$ of FasL and $\mathrm{IC}_{10} / \mathrm{IC}_{\mathrm{scr}}$ birinapant in comparison to mono treatment with FasL $(1 \mathrm{ng} / \mu \mathrm{l})$, and demonstrated synergistic effects when exposed to all concentrations $(1-50 \mathrm{ng} / \mu \mathrm{l})$ of FasL and $\mathrm{IC}_{10} / \mathrm{IC}_{\text {scr }}$ birinapant, which was also consistent with the results in the Annexin V assay (Fig. 3). Only PCI-1 and PCI-13 cells exhibited additive effects at the highest combination concentration (50 $\mathrm{ng} / \mu \mathrm{l} \mathrm{FasL}$ and $\mathrm{IC}_{10} / \mathrm{IC}_{\mathrm{scr}}$ birinapant). The HaCaT keratinocyte cell line exhibited slight effects in response to FasL mono treatment, and additive effects were observed in response to combination treatment. Significant $(\mathrm{p}<0.01)$ differences in cell number were observed in $\mathrm{HaCaT}$ at the concentrations of 12 and $25 \mathrm{ng} / \mu \mathrm{l}$ FasL and $\mathrm{IC}_{10} / \mathrm{IC}_{\mathrm{scr}}$ birinapant in comparison to mono treatment with FasL (12 and $25 \mathrm{ng} / \mu \mathrm{l})$.

\section{Discussion}

The present study suggested an important role for apoptosis sensitization with SMAC mimetics in the treatment of HNSCC cell lines. One of the main aims of HNSCC treatment is to induce apoptosis with an adjuvant concurrent high-dose platinum-based chemotherapy with single-fraction radiation (18). A characteristic of all cancer cells is their ability to evade apoptotic death pathways (5). The most common evasion mechanism is through the loss of the apoptosis control protein, p53, which is mutated or absent in half of all types of cancer (19). Similarly, proteins produced by the human papilloma virus (e.g., E6) bind to p53 to suppress its function (19). Cancer cells also overexpress anti-apoptotic proteins, including members of the IAP family, which are often deregulated or overexpressed in HNSCC $(20,21)$. Activation of the apoptotic pathway induces the mitochondrial outer membrane to release SMAC into the cytosol. Following binding to the corresponding IAPs, a pro-apoptotic effect is caused by the degradation of proteins by proteasomes $(22,23)$. Thus far, several monovalent and bivalent SMAC mimetics have entered clinical testing for dose escalation, mono therapy or combination therapy with chemotherapy and/or radiation (12,24-26).

To induce apoptosis via the extrinsic pathway, in the present study, it was first ensured that the corresponding
Table II. $\mathrm{IC}_{10}-\mathrm{IC}_{\mathrm{scr}}$ concentrations of birinapant.

\begin{tabular}{lcc}
\hline Cell line & $\mathrm{IC}_{10}(\mu \mathrm{M})$ & $\mathrm{IC}_{\text {scr }}(\mu \mathrm{M})$ \\
\hline PCI-1 & 18.2 & \\
PCI-9 & 1.3 & \\
PCI-13 & 11.5 & \\
PCI-52 & - & 100 \\
PCI-68 & - & 25 \\
Detroit 562 & - & 50 \\
FaDu & - & 25 \\
SCC9 & - & 1.6 \\
SCC25 & - & 1.6 \\
HaCaT & - & 1.6
\end{tabular}

${ }^{\mathrm{a}}$ For cell lines for which no $\mathrm{IC}_{10}$ value could be calculated based on dose-response curves, the concentration leading to the first significant cell count reduction was defined as the $\mathrm{IC}_{\mathrm{scr}}(\mu \mathrm{M})$ and used for combination treatment.

receptor was expressed in each cell line examined. Following the activation of the extrinsic pathway using FasL, a majority of the cell lines (PCI-52, PCI-68, Detroit 562, FaDu, SCC9 and SCC25) were not responsive to this treatment. After incubating the cell lines with birinapant as a single agent, each cell line exhibited an increase in cell death in the Annexin V assay. Notably, when exposed to combination treatment (FasL and birinapant $\mathrm{IC}_{10} / \mathrm{IC}_{\text {scr }}$ ), four of the former six FasL-resistant cell lines (PCI-68, Detroit 562, FaDu and SCC25) were sensitive to treatment and exhibited synergistic effects, while the effect of combination treatment was additive and synergistic with respect to the different concentrations for four (PCI-1, PCI-9, PCI-13 and HaCaT) of the previously sensitive cell lines. However, the concentrations of birinapant used could be limiting with regards to a potential prospective application in vivo. Only two cell lines (PCI-52 and SCC9) did not respond to combination treatment with FasL and birinapant. For SCC9, it is likely that cIAP1 or cIAP2 was not affected by birinapant, resulting in further inhibition of apoptosis. For the cell line PCI-52, a previous study by the authors demonstrated the degradation of cIAP1 through western blot analysis (15). However, a high concentration of birinapant (Table II) was used to observe minor effects of mono treatment on the cell line PCI-52 in the Annexin V assay. Therefore, other anti-apoptotic proteins may serve important roles in resisting cell death in these cell lines. According to Khan et al (27), survivin, another member of the IAP family, which contributes to radioresistance in HNSCC, is associated with poor outcomes in multiple types of cancer (28) and this protein may affect the ongoing chemoresistance in cancer cell lines. In support of this hypothesis, data from the same research group revealed the downregulation of survivin by oxaliplatin, resulting in enhanced radiosensitivity in HNSCC (27). Furthermore, silencing survivin suppressed cell proliferation and induced caspase-dependent apoptosis in HNSCC cells (29). Similarly, the previously identified endogenous inhibitor of apoptosis cellular FLICE-like inhibitory protein was implicated in caspase-dependent apoptosis in 
HNSCC cells (30) by promoting activation of the NF- $\kappa \mathrm{B}$ and extracellular signal-regulated kinase signaling pathways (31).

To the best of our knowledge, the present study is the first to demonstrate the additive and synergistic effects of combination therapy with FasL and birinapant for the treatment of HNSCC. Notably, seven of the nine HNSCC cell lines exhibited additive and/or synergistic effects when exposed to combination treatment, indicating a potentially major impact on HNSCC therapy.

\section{Acknowledgements}

The present study was supported by the Comprehensive Cancer Center Mainfranken and the Interdisciplinary Center for Clinical Research.

\section{References}

1. Kamangar F, Dores GM and Anderson WF: Patterns of cancer incidence, mortality, and prevalence across five continents: Defining priorities to reduce cancer disparities in different geographic regions of the world. J Clin Oncol 24: 2137-2150, 2006.

2. Torre LA, Bray F, Siegel RL, Ferlay J, Lortet-Tieulent J and Jemal A: Global cancer statistics, 2012. CA Cancer J Clin 65: 87-108, 2015.

3. Gupta S, Kong W, Peng Y, Miao Q and Mackillop WJ: Temporal trends in the incidence and survival of cancers of the upper aerodigestive tract in Ontario and the United States. Int J Cancer $125: 2159-2165,2009$.

4. Moreira J, Tobias A, O'Brien MP and Agulnik M: Targeted therapy in head and neck cancer: An update on current clinical developments in epidermal growth factor receptor-targeted therapy and immunotherapies. Drugs 77: 843-857, 2017.

5. Hanahan D and Weinberg RA: Hallmarks of cancer: The next generation. Cell 144: 646-674, 2017.

6. Fulda S: Promises and challenges of smac mimetics as cancer therapeutics. Clin Cancer Res 21: 5030-5036, 2015.

7. Derakhshan A, Chen Z and Van Waes C: Therapeutic small molecules target inhibitor of apoptosis proteins in cancers with deregulation of extrinsic and intrinsic cell death pathways. Clin Cancer Res 23: 1379-1387, 2017.

8. Johnstone RW, Ruefli AA and Lowe SW: Apoptosis: A link between cancer genetics and chemotherapy. Cell 108:153-164, 2002.

9. Yu J and Zhang L: Apoptosis in human cancer cells. Curr Opin Oncol 16: 19-24, 2004.

10. Sun Q, Zheng X, Zhang L and Yu J: Smac modulates chemosensitivity in head and neck cancer cells through the mitochondrial apoptotic pathway. Clin Cancer Res 17: 2361-2372, 2011.

11. Yang XH, Feng ZE, Yan M, Hanada S, Zuo H, Yang CZ, Han ZG, Guo W, Chen WT and Zhang P: XIAP is a predictor of cisplatin-based chemotherapy response and prognosis for patients with advanced head and neck cancer. PLoS One 7: e31601, 2012

12. Matzinger O, Viertl D, Tsoutsou P, Kadi L, Rigotti S, Zanna C, Wiedemann N, Vozenin MC, Vuagniaux G and Bourhis J: The radiosensitizing activity of the SMAC-mimetic, Debio 1143 , is TNF $\alpha$-mediated in head and neck squamous cell carcinoma. Radiother Oncol 116: 495-503, 2015.

13. Eytan DF, Snow GE, Carlson SG, Schiltz S, Chen Z and Van Waes C: Combination effects of SMAC mimetic birinapant with TNF $\alpha$, TRAIL, and docetaxel in preclinical models of HNSCC. Laryngoscope 125: E118-E124, 2015.

14. Medicine USNLo: ClinicalTrials.gov. U.S. National Library of Medicine, Rockville Pike, Bethesda, 2017. https://clinicaltrials. gov/ct $2 /$ results?cond $=$ solid $\&$ term $=$ smac $\&$ cntry $1=\&$ state $1=\&$ Sea rch=Search. Accessed August 7, 2017.
15. Brands RC, Herbst F, Hartmann S, Seher A, Linz C, Kübler AC and Müller-Richter UDA: Cytotoxic effects of SMAC-mimetic compound LCL161 in head and neck cancer cell lines. Clin Oral Investig 20: 2325-2332, 2016.

16. Brands RC, Muller-Richter UD, De Donno F, Seher A, Mutzbauer G, Linz C, Kübler AC and Hartmann S: Co-treatment of wild-type EGFR head and neck cancer cell lines with afatinib and cisplatin. Mol Med Rep 13: 2338-2344, 2016.

17. Eytan DF, Snow GE, Carlson S, Derakhshan A, Saleh A, Schiltz S, Cheng H, Mohan S, Cornelius S, Coupar J, et al: SMAC mimetic birinapant plus radiation eradicates human head and neck cancers with genomic amplifications of cell death genes FADD and BIRC2. Cancer Res 76: 5442-5454, 2016.

18. Adelstein DJ, Li Y, Adams GL, Wagner H Jr, Kish JA, Ensley JF, Schuller DE and Forastiere AA: An intergroup phase III comparison of standard radiation therapy and two schedules of concurrent chemoradiotherapy in patients with unresectable squamous cell head and neck cancer. J Clin Oncol 21: 92-98, 2003.

19. Samarasinghe B: Hallmarks of Cancer 3: Evading Apoptosis. Scientific American, 2013. https://blogs.scientificamerican.com/ guest-blog/hallmarks-of-cancer-3-evading-apoptosis/. Accessed August 7,2017.

20. De Maria S, Pannone G, Bufo P, Santoro A, Serpico R, Metafora S, Rubini C, Pasquali D, Papagerakis SM, Staibano S, et al: Survivin gene-expression and splicing isoforms in oral squamous cell carcinoma. J Cancer Res Clin Oncol 135: 107-116, 2009.

21. Khan Z and Bisen PS: Oncoapoptotic signaling and deregulated target genes in cancers: Special reference to oral cancer. Biochim Biophys Acta 1836: 123-145, 2013.

22. Chen DJ and Huerta S: Smac mimetics as new cancer therapeutics. Anticancer Drugs 20: 646-658, 2009.

23. Koff JL, Ramachandiran S and Bernal-Mizrachi L: A time to kill: Targeting apoptosis in cancer. Int J Mol Sci 16: 2942-2955, 2015.

24. Amaravadi RK, Schilder RJ, Martin LP, Levin M, Graham MA, Weng DE and Adjei AA: A phase I study of the SMAC-mimetic birinapant in adults with refractory solid tumors or lymphoma. Mol Cancer Ther 14: 2569-2675, 2015.

25. Infante JR, Dees EC, Olszanski AJ, Dhuria SV, Sen S, Cameron S and Cohen RB: Phase I dose-escalation study of LCL161, an oral inhibitor of apoptosis proteins inhibitor, in patients with advanced solid tumors. J Clin Oncol 32: 3103-3110, 2014.

26. Tolcher AW, Bendell JC, Papadopoulos KP, Burris HA, Patnaik A, Fairbrother WJ, Wong H, Budha N, Darbonne WC, Peale F, et al: A phase I dose-escalation study evaluating the safety tolerability and pharmacokinetics of CUDC-427, a potent, oral, monovalent IAP antagonist, in patients with refractory solid tumors. Clin Cancer Res 22 : 4567-4573, 2016.

27. Khan Z, Khan N, Tiwari RP, Patro IK, Prasad GB and Bisen PS: Down-regulation of survivin by oxaliplatin diminishes radioresistance of head and neck squamous carcinoma cells. Radiother Oncol 96: 267-273, 2010.

28. Qi G, Kudo Y, Ando T, Tsunematsu T, Shimizu N, Siriwardena SB, Yoshida M, Keikhaee MR, Ogawa I and Takata T: Nuclear Survivin expression is correlated with malignant behaviors of head and neck cancer together with Aurora-B. Oral Oncol 46: 263-270, 2010

29. Khan Z, Tiwari RP, Khan N, Prasad GB and Bisen PS: Induction of apoptosis and sensitization of head and neck squamous carcinoma cells to cisplatin by targeting survivin gene expression. Curr Gene Ther 12: 444-53, 2012.

30. Irmler M, Thome M, Hahne M, Schneider P, Hofmann K, Steiner V, Bodmer JL, Schröter M, Burns K, Mattmann C, et al: Inhibition of death receptor signals by cellular FLIP. Nature 388: 190-195, 1997

31. Kataoka T, Budd RC, Holler N, Thome M, Martinon F, Irmler M, Burns K, Hahne M, Kennedy N, Kovacsovics M and Tschopp J: The caspase-8 inhibitor FLIP promotes activation of NF-kappaB and Erk signaling pathways. Curr Biol 10: 640-648, 2000. 